\title{
XXXIV. Integration theorems of four-dimensional vector analysis
}

\section{H.T. Flint}

To cite this article: H.T. Flint (1921) XXXIV. Integration theorems of four-dimensional vector analysis, Philosophical Magazine Series 6, 41:243, 389-404, DOI: 10.1080/14786442108636230

To link to this article: http://dx.doi.org/10.1080/14786442108636230

曲 Published online: 08 Apr 2009.

Submit your article to this journal ๘

Џ Article views: 2

Q View related articles ¿ 
XXXIV. Integration Theorems of Four-Dimensional Vector Analysis. By H. T. FLINT, Lecturer in Physics, King's College, London *.

\section{Introduction.}

TWE four-dimensional rector analysis developed by Sommerfeld (Ann. der Physik, vols. xxxii. p. 749 sqq. and xxxiii. p. 649 sqq.) evidently bears the same relation to a more generul analysis as do scalar and vector products and the theorems of Gauss and Stokes to vector or quaternion analysis.

In attempting to work out some of the details of the restricted principle of Relativity in terms of the more general notation, it was observed that such expressions as the scalar and vector products of four vectors, combinations of four and six vectors, together with the generalized div and curl and the operation lor (Lorentz operator) of Minkowski's Calculus are parts of more general expressions.

It is the object of the following pages to set forth this analysis. It will be obvious to the reader how much is owed to quaternion analysis, in particular to Joly's 'Manual of Quaternions,' ch. xviii., of which the notation is adopted here, and to Sommerfeld's papers mentioned above. The application of the notation to the theory of Relativity has already been pointed out by Professor Johnston and discussed by Sir J. Larmor $\dagger$.

In the first few sections the notation is explained before the main object of developing the integral theorem is reached.

$\S 1$. In treating space of four dimensions it is usual and convenient to adopt a language and notation similar to that with which we are familiar in three, though it is difficult and perhaps impossible to form corresponding geometrical pictures.

Thus a straight line through the points $\left(a_{1} b_{1} c_{1} d_{1}\right)$ $\left(a_{2} \dot{b}_{2} c_{9} d_{2}\right)$ is the locus :

$$
\frac{x-a_{1}}{a_{1}-a_{2}}=\frac{y-b_{1}}{b_{1}-b_{2}}=\frac{z-c_{1}}{c_{1}-c_{2}}=\frac{u-d_{1}}{d_{1}-d_{2}}
$$

$(x y z u)$ being current coordinates, axes $\mathrm{O} x, \mathrm{O} y, \mathrm{O} z, \mathrm{O} u$ beiug assumed as axes of reference.

* Communicated by the Author.

+ Proc. Roy. Soc. Dec. 1919.

Phil. Mag. S. 6. Vol. 41. No. 243. Mareh 1921. 2 D 
A vector is a quantity which may be represented by a straight line in this way

We assume four directions in space and denote them by $i_{1} i_{2} i_{3} i_{1}$ and any other vector $\rho$ may then be expressed in terms of them :

$$
\rho=x i_{1}+y i_{2}+z i_{3}+u i_{4} .
$$

If $i_{1} i_{2} i_{3} i_{4}$ are unit vectors, $x y z u$ are the projections of $\rho$ along them.

$\S 2$. If two vectors $\rho_{1}$ and $\rho_{2}$ are multiplied an expression results containing terms in $i_{m} i_{n}$ and $i_{m} i_{m}$, where $m \neq n$.

We define the product of the two like vectors $i_{m} i_{m}$ to be -1 , and the product of $\rho_{1} \rho_{2}$ then contains terms in two vectors and other terms from which the $i$ 's have disappeared.

We write :

$$
\rho_{1} \rho_{2}=\mathrm{V}_{2} \rho_{1} \rho_{2}+\mathrm{V}_{0} \rho_{1} \rho_{2} . \quad \text {. . . . . }
$$

$\mathrm{V}_{2} \rho_{1} \rho_{2}$ contains $i_{2} i_{3}, i_{1} i_{2}$ etc. and $\mathrm{V}_{0} \rho_{1} \rho_{2}$ denotes the remainder of the product not containing any $i$ s. $\mathrm{V}_{0} \rho_{1} \rho_{2}$ with a minus sign prefixed is called the scalar product of $\rho_{1} \rho_{2}$.

If this vanishes $\rho_{1}$ and $\rho_{2}$ are said to be perpendicular. We complete our definition of the product $\rho_{1} \rho_{2}$ by writing

$$
\rho_{2} \rho_{1}=-V_{2} \rho_{1} \rho_{2}+V_{0} \rho_{1} \rho_{2} . . . . .
$$

'This gives a rule of multiplication known in the case of ordinary vector analysis. It is not commutative but is defined to be associative and distributive.

In choosing the unit vectors $i_{1} i_{2} i_{3} i_{4}$ as fundamental directions we make them mutually perpendicular,

$$
\text { i. e. } \quad V_{0} i_{2} i_{3}=0 \text { ete. }
$$

The rule of multiplication then sbows that

If we write :

$$
i_{m} i_{n}=-i_{n} i_{m}, \quad m \neq n .
$$

$$
\left.\begin{array}{l}
\rho_{1}=a_{1} i_{1}+b_{1} i_{2}+c_{1} i_{3}+d_{1} i_{4}, \\
\rho_{2}=a_{2} i_{1}+b_{2} i_{2}+c_{2} i_{3}+d_{2} i_{4}
\end{array}\right\} \quad . .
$$

we have on expansion : and

$$
V_{0} \rho_{1} \rho_{2}=-\left(a_{1} a_{2}+b_{1} b_{2}+c_{1} c_{2}+d_{1} d_{2}\right)
$$

$$
\begin{aligned}
\mathrm{V}_{2} \rho_{1} \rho_{2} & =i_{2} i_{3}\left(b_{1} c_{2}-b_{2} c_{1}\right)+i_{3} i_{1}\left(c_{1} a_{2}-c_{2} a_{1}\right)+i_{1} i_{2}\left(a_{1} b_{2}-a_{2} b_{1}\right) \\
& +i_{1} i_{4}\left(a_{1} d_{2}-a_{2} d_{1}\right)+i_{2} i_{4}\left(b_{1} d_{2}-b_{2} d_{1}\right)+i_{3} i_{4}\left(c_{1} d_{2}-c_{2} d_{1}\right) .
\end{aligned}
$$


The vector $V_{2} \rho_{1} \rho_{2}$ is the vector area of the parallelogram of which the two adjacent sides are $\rho_{1}$ and $\rho_{2}$. It can be split up into six components, which we may denote by $\mathrm{A}_{y z}, \mathrm{~A}_{z x}$, etc, these being the coefficients of $i_{2} i_{3}, i_{1} i_{2}$, etc., and which are the projections of the area on the six planes which may be chosen out of the four fundamental directions. The vector area although it has six coefficients is defined by five quantities, for a relation exists betwoen the coefficients, viz. :

$$
\mathrm{A}_{y z} \mathrm{~A}_{x u}+\mathrm{A}_{z x} \mathrm{~A}_{y u}+\mathrm{A}_{x y} \mathrm{~A}_{z u}=0, \quad . \quad .
$$

as can be seen by expansion of the determinants.

The elementary area bounded by two short lines $d \rho$ and $\delta \rho$ may be written :

$$
i_{2} i_{\varepsilon}(d y \delta z-\delta y d z)+i_{3} i_{1}(d z \delta x-\delta z d x)+\ldots .
$$

Minkowski uses the terms four- and six-vectors in a particular sense, the vectors are subject to a particular transformation, but the comparison of these vectors with $(2 \cdot 3)$ and $(2 \cdot 4)$ is clear, e.g. $(2 \cdot 4)$ is a six-vector without the limitations regarding the transformation.

'Thus the scalar and vector products of Minkowski's calculus are component parts of the complete quaternionic product $\rho_{1} \rho_{2}$. We may not speak of a unique perpendicular to a plane in four dimensions, for just as in three dimensions a perpendicular to a line is not determinate, so in three the perpendicular to a plane is not definite.

We have seen that a plane may be defined by two lines $\alpha$ and $\beta$, and it is denoted by $\nabla_{2} \alpha \beta$.

The magnitude of the area, or the tensor as it is called, is defined to be the square root of the expression :

$$
A_{y z}{ }^{2}+A_{z x}{ }^{2}+A_{x y}{ }^{2}+A_{z u}{ }^{2}+A_{y u}{ }^{2}+A_{z u}{ }^{2} \text {. }
$$

If this is unity the plane is a unit plane.

We may now show that the plane normal to $\mathrm{V}_{2} \alpha \beta$ is definite if we define it as the locus of lines perpendicular both to $a$ and to $\beta$ and consequently to any line in their plane. For let the unit plane normal to $\mathrm{V}_{2} \propto \beta$ be $\mathrm{V}_{2} \gamma \delta$.

Then by definition :

$$
\mathrm{V}_{0} \alpha \gamma=\mathrm{V}_{0} \alpha \delta=\mathrm{V}_{0} \beta \gamma=\mathrm{V}_{0} \beta \delta=0,
$$

and the tensor of $V_{2} \gamma \delta=1$

$$
\text { or } \mathrm{T} \mathrm{V}_{2} \gamma \delta=1 \text {. }
$$


These five equations determine the five quantities necessary to fix the plane, which is therefore definite if $V_{2} x \beta$ is known.

It is easy to show that $V_{2} \gamma \delta$ written in full is :

$$
i_{2} i_{3} \mathrm{~A}_{x u}+i_{3} i_{1} \mathrm{~A}_{y u}+i_{1} i_{2} \mathrm{~A}_{z u}+i_{1} i_{4} \mathrm{~A}_{y z}+i_{2} i_{4} \mathrm{~A}_{z x}+i_{3} i_{4} \mathrm{~A}_{x y}
$$

where the $A$ 's denote the components of the original plane. It is to be noted that the coefficients $A_{y z}$ and $A_{x u}$, and similarly for the other pairs, change their places in passing from a plane to its normal. We have tacitly assumed in this expansion of $V_{2} \gamma \delta$ that $V_{2} \times \beta$ is also a unit plane. If it is not, we have only to multiply thronghout by a constant-the resiprocal of the tensor.

Thus if a plane is represented by $k V_{2}$, where $k$ is a scalar constant, the tensor of the plane and $V_{2}$ is the unit vector defining it vectorially, any plane normal to it is $k_{i}^{\prime} V_{2}{ }^{\prime}$, where $k^{\prime}$ is the tensor of the latter and $V_{2}^{\prime}$ is obtained from $V_{2}$ by interchanging the coefficients in the above manner.

Functions enter into analysis which have the structure of a vector area but do not satisfy the condition $(2 \cdot 5)$. They are defined by $b$ quantities, and we may write such a function as

$$
\mathrm{P}_{2}=i_{2} i_{3} \mathrm{P}_{y z}+i_{3} i_{1} \mathrm{P}_{z x}+i_{1} i_{2} \mathrm{P}_{x y}+i_{1} i_{4} \mathrm{P}_{x u}+i_{3} i_{4} \mathrm{P}_{y u}+i_{3} i_{1} \mathrm{P}_{z u} \text {, }
$$

and there corresponds to this the reciprocal function :

$$
\mathbf{P}_{2}^{\prime}=i_{2} i_{3} \mathrm{P}_{x u}+\ldots+\ldots+i_{\mathbf{1}} i_{4} \mathrm{P}^{y z}+\ldots+\ldots
$$

But in this case the relation

$$
\mathrm{V}_{2} \mathrm{P}_{2} \mathrm{P}_{2}^{\prime} \neq 0
$$

as in the case of the product of vector areas when these are normal.

We shall return to this point later.

It is to be noted that we can always express $P_{2}$ in the form :

$$
\mathrm{P}_{2}=k V_{2} \alpha \beta+k^{\prime} V_{2}^{\prime} \alpha \beta \text {. }
$$

$k^{\prime}$ gives the additional arbitrary constant.

This should be compared with Sommerfeld's discussion, $\S 1$ of vol xxxii., already referred to.

If a vector area be split up into elements so that each element projects into rectangles on the coordinate planes, we write for it:

$$
\begin{aligned}
d \nu=i_{2} i_{3} d y d z & +i_{2} i_{1} d z d x+i_{1} i_{2} d x d y \\
& +i_{1} i_{4} d x d u+i_{2} i_{4} d y d u+i_{3} i_{4} d z d u . .
\end{aligned}
$$

This element is a vector like $\mathrm{P}_{2}$. 
$\S 3$. The laws of multiplication lead to certain relations between products of the $i$ 's of which the following are illustrative :

$$
\left.\begin{array}{l}
i_{1} i_{2} i_{1}=-i_{1} i_{1} i_{2}=i_{2} \\
i_{2} i_{2} i_{1}=-i_{1}
\end{array}\right\}
$$

A change of position of amy vector by an odd number of places in any product changes the sign, unless the product is scalar, or contains a scalar part.

Thus

$$
\alpha \beta \gamma=-\beta \alpha \gamma=\beta \gamma \alpha .
$$

Products of the type $i_{1} i_{2} i_{3}$ are not reducible.

In multiplying three vectors it is convenient to regard the product is composed of reducible and irredneible parts. Thus

$$
\rho_{1} \rho_{2} \rho_{3}=V_{8} \rho_{1} \rho_{2} \rho_{3}+V_{1} \rho_{1} \rho_{2} \rho_{3} ; \quad . \quad . \quad .
$$

$V_{3} \rho_{1} \rho_{2} \rho_{3}$ contains terms in $i_{2} i_{3} i_{4}, i_{3} i_{4} i_{1}$, etc., and $V_{1} \rho_{1} \rho_{2} \rho_{3}$ contains terms in $i_{1} i_{2} i_{3}$ and $i_{4}$ which are the result of such reduction as is illustrated in $(3 \cdot 1)$.

If $\rho_{1}$ and $\rho_{2}$ have the values in $(2 \cdot 3)$ and

$$
\rho_{3}=a_{3} i_{1}+b_{3} i_{2}+c_{3} i_{3}+d_{3} i_{4}
$$

the expansion of the product $V_{3} \rho_{1} \rho_{2} \rho_{3}$ gives:

$$
\begin{aligned}
\nabla_{2} \rho_{1} \rho_{2} \rho_{3}= & i_{2} i_{3} i_{4}\left(b_{1} c_{2} d_{3}\right)+i_{3} i_{4} i_{1}\left(c_{1} d_{2} a_{2}\right) \\
& +\left(i_{4} i_{1} i_{2}\right)\left(d_{1} a_{2} b_{3}\right)+i_{1} i_{2} i_{3}\left(a_{1} b_{2} c_{3}\right), \quad . \quad .
\end{aligned}
$$

where

$$
\left(\mathrm{A}_{1} \mathrm{~B}_{2} \mathrm{C}_{3}\right) \equiv\left|\begin{array}{ccc}
\mathrm{A}_{1} & \mathrm{~B}_{1} & \mathrm{C}_{1} \\
\mathrm{~A}_{2} & \mathrm{~B}_{2} & \mathrm{C}_{2} \\
\mathrm{~A}_{3} & \mathrm{~B}_{3} & \mathrm{C}_{3}
\end{array}\right|
$$

The determinants are recognized as three-dimensional volumes. They are the components of the directed volume $\mathrm{V}_{3} \rho_{1} \rho_{2} \rho_{3}$ on the various sets of three dimensions that may be chosen from the four.

The coefficients of these irreducible products are minors of the determinant $\left(a_{1} b_{2} c_{3} d_{4}\right)$. and in writing the components $V_{1} V_{2} V_{3} V_{4}$ we shall adopt the rule of signs:

$$
\begin{array}{ll}
\mathrm{V}_{1}=\left(b_{1} c_{2} d_{3}\right), & \mathrm{V}_{2}=-\left(c_{1} d_{2} a_{3}\right), \quad \mathrm{V}_{3}=\left(d_{1} a_{2} b_{3}\right), \\
\mathrm{V}_{4}=-\left(a_{1} b_{2} c_{3}\right) . &
\end{array}
$$


Thus for any directed volume $\mathrm{V}$ we have :

$$
\mathrm{V}=i_{2} i_{3} i_{4} V_{1}-i_{3} i_{4} i_{1} V_{2}+i_{4} i_{1} i_{2} V_{3}-i_{1} i_{2} i_{3} V_{4}
$$

The volume element $d \tau$ may be divided into elementary components. So that:

$$
d \tau=i_{2} i_{3} i_{4} d z d y d u-i_{3} i_{4} i_{1} d y d u d u+i_{4} i_{1} i_{2} d u d x d y-i_{1} i_{2} i_{3} d x d y d z \text {. }
$$

The remaining part of the complete product $\rho_{1} \rho_{2} \rho_{3}$ is $V_{1} \rho_{1} \rho_{2} \rho_{3}$, and on expansion it is seen to be :

$-i_{1} a_{1} \Sigma a_{2} a_{3}+i_{1}\left\{b_{1}\left(a_{2} b_{3}\right)+c_{1}\left(a_{2} c_{3}\right)+d_{1}\left(a_{2} d_{3}\right)\right\}+$ similar terms.

The coefficient of the second $i_{1}$ is the $O X$ component of the vector product of a four- and six-vector employed in Minkowski's Calculus. It may be represented in our notation as :

$$
\mathrm{V}_{1}\left(\rho_{1} \mathrm{~V}_{2} \rho_{2} \rho_{3}\right) \cdot \text {. . . . . }
$$

$\$ 4$. The product of four vectors will consist of terms in $i_{1} i_{2} i_{3} i_{4}$, others in $i_{2} i_{3}$ which have arisen from such products as $i_{2} i_{4} i_{3} i_{4}$ and so on, and finally there will be terms independent of $i$ 's arising from such products as $i_{2} i_{3} i_{2} i_{3}$ which reduce to

Thus

$$
i_{2} i_{3} i_{2} i_{3}=-i_{2} i_{2} i_{3} i_{3}=-1 \text {. }
$$

$$
\rho_{1} \rho_{2} \rho_{3} \rho_{4}=V_{4} \rho_{1} \rho_{2} \rho_{3} \rho_{4}+V_{2} \rho_{1} \rho_{2} \rho_{3} \rho_{4}+V_{0} \rho_{1} \rho_{2} \rho_{3} \rho_{4} .
$$

On expansion we find :

$$
V_{4} \rho_{1} \rho_{2} \rho_{3} \rho_{4}=i_{1} i_{2} i_{3} i_{4}\left(a_{1} b_{2} c_{3} d_{4}\right) . \quad . \quad .
$$

Thus the tensor of $V_{4} \rho_{1} \rho_{2} \rho_{3} \rho_{4}$, or $\mathrm{TV}_{4} \rho_{1} \rho_{2} \rho_{3} \rho_{4}$ as it will be written, is the volume of the four-dimensional parallelepiped bounded by the four vectors.

It is not possible to write $i_{1} i_{2} i_{3} i_{4}= \pm 1$ by analogy with the corresponding $i j k=-1$, for this would lead to inconsistencies.

Suppose we write $\quad i_{1} i_{2} i_{3} i_{4}=-1$.

Then

$$
i_{1} i_{1} i_{2} i_{3} i_{4}=-i_{1}
$$

and

$$
i_{1} i_{2} i_{3} i_{4} i_{1}=-i_{1} \text {. }
$$

The first gives:

$$
\begin{aligned}
& i_{2} i_{3} i_{4}=i_{1}, \\
& i_{2} i_{3} i_{4}=-i_{1} .
\end{aligned}
$$

the second 
Again we should have :

$$
\text { or } \quad \begin{aligned}
i_{1} i_{4} i_{1} i_{2} i_{3} i_{4} & =-i_{1} i_{4} \\
i_{2} i_{3} & =i_{1} i_{4} .
\end{aligned}
$$

The product $i_{1} i_{2} i_{3} i_{4}$ will be denoted by $\mathrm{I}$.

When I enters into any expression it obeys the ordinary rule of sign, i.e. an odd number of displacements of $I$ in a vector product changes the sign, while an even number leaves the sign unchanged.

$$
\mathrm{I} \alpha \beta \gamma=-\alpha \mathrm{I} \beta \gamma=\alpha \beta \mathrm{I} \gamma=-\alpha \beta \gamma \mathrm{I} \text {. }
$$

Also

$$
\mathrm{I} i_{2} i_{3}=-i_{1} i_{4}
$$

and

$$
\mathrm{I} i_{2} i_{3} i_{4}=i_{1} \text { etc. }
$$

Thus the factor $-I$ will change a product $V_{2}$ into its reciprocal and $V_{3}$ is changed into a vector of the type $V_{3}$.

We have evidently

$$
I V_{s} \alpha \beta \gamma=V_{I} I \alpha \beta \gamma
$$

$\S 5$. The definition of the component of a vector $\rho$ along any direction denoted by the unit vector $n$ is similar to that in ordinary vector analysis. We define the component to be $-\mathrm{V}_{0} \rho n$.

In the case of a plane we define the component in the plane of a vector of the type $V_{2}$ in a similar way.

The component is $-\mathrm{V}_{0} \mathrm{P}_{2} \nu$, where $\nu$ denotes a unit plane.

Thus the component of $\mathrm{P}_{2}$ in the plane $y z$ is

$$
-\mathrm{V}_{0} \mathrm{P}_{2} i_{2} i_{3}=\mathrm{P}_{y z} \text {. }
$$

We cannot define a component along a vector normal to the plane since the normal is indefinite; we may, however, define a component in the reciprocal plane.

Let $V_{2} \alpha \beta$ denote a unit plane, and $V_{2}{ }^{\prime} \alpha \beta$ its reciprocal. The component normal to $V_{2}$ may be defined as

$$
-\mathrm{V}_{0} \mathrm{P}_{2} \mathrm{~V}_{2}^{\prime} \alpha \beta
$$

or what is the same thing :

$$
\mathrm{V}_{0} \mathrm{P}_{2}\left(\mathrm{IV}_{2} \alpha \beta\right) \text {. }
$$

This is equal to $\mathrm{V}_{0} \mathrm{IP}_{2} \mathrm{~V}_{2} \alpha \beta=-\mathrm{V}_{0} \mathrm{P}_{2}^{\prime} \mathrm{V}_{2} \alpha \beta$.

Thus the component of a vector in the reciprocal (or normal) plane is equal to the component of the reciprocal $n$ the plane. 
It is to be noted that $V_{0}\left(V_{2} \alpha \beta\right)\left(V_{2}{ }^{\prime} \alpha \beta\right)=0$ on account of the relation $(2 \cdot 5)$, so that the two planes may be said to be normal in the same way that two vectors $\rho_{1}$ and $\rho_{2}$ are normal if $\mathrm{V}_{0} \rho_{1} \rho_{2}=0$.

But

$$
\begin{array}{ll}
\mathrm{V}_{0} \mathrm{P}_{2} \mathrm{P}_{2}^{\prime} \neq 0 & \text { althongh the relation } \\
\mathrm{V}_{0} \mathrm{P}_{2} \mathrm{P}_{2}^{\prime}=\mathrm{V}_{0} \mathrm{P}_{2}^{\prime} \mathrm{P}_{2} & \text { still holds. }
\end{array}
$$

Sommerfeld has defined the components of a six-vector with respect to a plane. We can very easily obtain his result in our notation. The component is $-V_{0} P_{2}\left(V_{2} \alpha \beta\right)$.

We must make $V_{2} \alpha \beta$ a unit plane and naturally shall choose $\alpha$ and $\beta$ as two unit perpendicular vectors.

Write

$$
\begin{aligned}
& \alpha=l_{1} i_{1}+m_{1} i_{2}+n_{1} i_{3}+p_{1} i_{4}, \\
& \beta=l_{2} i_{1}+m_{2} i_{2}+n_{2} i_{3}+p_{2} i_{4} .
\end{aligned}
$$

Since $\alpha$ and $\beta$ are unit perpendicular vectors,

$$
\text { and } \begin{aligned}
\Sigma l_{1}{ }^{2}=\Sigma l_{2}{ }^{2} & =1 \\
\Sigma l_{1} l_{2} & =0 .
\end{aligned}
$$

Then $-\mathrm{V}_{0} \mathrm{P}_{2} \mathrm{~V}_{2} \alpha \beta$

$$
\begin{aligned}
& =-\nabla_{0}\left(\sum i_{2} i_{3} \mathrm{P}_{y z}\right)\left(\Sigma i_{2} i_{3}\left(m_{1} n_{2}\right)\right) \\
& =\mathrm{P}_{y z}\left(m_{1} n_{2}\right)+\mathrm{P}_{z x}\left(n_{1} l_{2}\right)+\mathrm{P}_{x y}\left(l_{1} m_{2}\right)+\mathrm{P}_{x u}\left(l_{1} p_{2}\right) \\
& \\
& \quad+\mathrm{P}_{y u}\left(m_{1} p_{2}\right)+\mathrm{P}_{z u}\left(n_{1} p_{2}\right) .
\end{aligned}
$$

This should be compared with Sommerfeld's result ( $A n n$. d. Phys. xxxii. p. 760).

For a vector $A$ of the directed volume type we have:

$$
\begin{aligned}
\mathrm{A}_{3} & =i_{2} i_{3} i_{4} \mathrm{~A}_{x}-i_{3} i_{4} i_{1} \mathrm{~A}_{y}+i_{4} i_{1} i_{2} \mathrm{~A}_{z}-i_{1} i_{2} i_{3} \mathrm{~A}_{u} \\
& =\mathrm{I}\left(i_{1} \mathrm{~A}_{x}+i_{2} \mathrm{~A}_{y}+i_{3} \mathrm{~A}_{z}+i_{4} \mathrm{~A}_{u}\right)=\mathrm{I} \mathrm{A}_{1} \text { (say). }
\end{aligned}
$$

The component of $\mathrm{A}$ along any direction $n$ may be written :

$$
-\mathrm{TV}_{4} \mathrm{~A}_{3} n \text { or }-\mathrm{V}_{0}\left(\mathrm{IA}_{3}\right) n \text { or }-\mathrm{V}_{0} \mathrm{~A}_{1} n \text {. }
$$

The component of $A_{3}$ along $O x$ is by this definition $A_{x}$, and similarly $A_{y} A_{z} A_{u}$ are the components along the other axes.

$A_{3}$ is normal to any direction $n$ if $\mathrm{V}_{0}\left(\mathrm{IA}_{3}\right) n=0$.

If four vectors $\rho_{1} \rho_{2} \rho_{3} \rho_{4}$ bound a four-dimensional volume 
it is convenient to adopt a rule of signs for the three-dimensional volumes that may be chosen from them. The rule adopted is to define the volumes as :

$$
+\mathrm{V}_{3} \rho_{2} \rho_{3} \rho_{4}, \quad-\mathrm{V}_{3} \rho_{3} \rho_{4} \rho_{1}, \quad+V_{3,} \rho_{1} \rho_{1} \rho_{2}, \quad-V_{3} \rho_{1} \rho_{2} \rho_{3} .
$$

This is to be associated with the rule of $\$ 3$. The volumes corresponding to the vectors $i_{1} d x, i_{2} d y, i_{3} d z, i_{4} d u$ are :

$$
\begin{gathered}
i_{2} i_{3} i_{4} d y d z d u, \quad-i_{3} i_{4} i_{1} d z d u d x, \quad i_{4} i_{1} i_{2} d u d x d y, \\
\text { and }-i_{1} i_{2} i_{3} d x d y d z .
\end{gathered}
$$

$\S 6$. A vector operator $D_{1}$ defined as

$$
i_{1} \frac{\partial}{\partial x}+i_{2} \frac{\partial}{\partial y}+i_{3} \frac{\partial}{\partial z}+i_{4} \frac{\partial}{\partial u}
$$

plays an important part in four-vector analysis just as the Hamiltonian $\nabla$ does in three dimensions.

The suffix $D_{1}$ serves to show that it is of the type of a vector $V_{1}$.

Any function $\sigma$ of $\rho$ will undergo a small change $d \sigma$, on account of a variation $d \phi$ given by the equation

$$
d \sigma=-\mathrm{V}_{0} d \rho \mathrm{D}_{1}, \sigma . \quad . \quad . \quad .
$$

$(6 \cdot 1)$ is the same result as is expressed in Cartesians by :

$$
d \sigma=\frac{\partial \sigma}{\partial x} d x+\frac{\partial \sigma}{\partial y} d y+\frac{\partial \sigma}{\partial z} d z+\frac{\partial \sigma}{\partial u} \cdot d u .
$$

This method of introducing $\mathrm{D}_{1}$ appears to be the simplest. $\nabla$ is sometimes defined by the operation :

$$
\operatorname{Lim}_{v \rightarrow 0} \frac{\int d v \cdot F(\rho)}{v}=\nabla \mathrm{F}(\rho)
$$

where $d \nu$ denotes a divided element of area in two dimensions bounding a small volume $v$, and $F$ is any function of $\rho$, the vector from the origin to $d v$.

If this method of defining an operator be extended to four dimensions we shall have :

$$
\operatorname{Lim}_{v \rightarrow 0} \frac{\int d \tau \mathrm{F}(\rho)}{v}=\operatorname{DF}(\rho),
$$

where $d \tau$ is the directed element of three-dimensional volume and $v$ a small four-dimensional volume. 
This mode of arriving at $\mathrm{D}$ gives to it the inconvenient canonical form :

$$
i_{2} i_{3} i_{4} \frac{\partial}{\partial x}-i_{3} i_{4} i_{1} \frac{\partial}{\partial y}+i_{4} i_{1} i_{2} \frac{\partial}{\partial z}-i_{1} i_{2} i_{3} \frac{\partial}{\partial u} .
$$

$\S 7$. The circuital theorems of ordinary vector analysis

$$
\int d \rho \mathrm{F}(\rho)=\iint \mathrm{V}(d \nu \nabla) \cdot \mathrm{F}(\rho)
$$

and

$$
\iint d \nu \mathrm{F}(\rho)=\iiint \nabla \mathrm{F}(\rho) \cdot d \nu
$$

are generalizations of Stokes's and Gauss's theorems. We proceed to establish the corresponding circuital theorems for four-vectors. They are three in number and the operator $\nabla$ is now replaced by $D_{1}$.

\&8. In order to establish the theorem for integration along a line we require the relation:

$$
\alpha_{1} V_{0} \alpha_{2} \alpha-\alpha_{2} V_{0} \alpha_{1} \alpha=\nabla_{1}\left(V_{2} \alpha_{1} \alpha_{2}, \alpha\right) . \quad .
$$

If we write : $\quad \alpha_{1}=i_{1} \alpha_{1}+i_{2} b_{1}+i_{3} c_{1}+i_{4} d_{1}$,

$$
\begin{aligned}
& \alpha_{2}=i_{1} a_{2}+i_{2} b_{2}+i_{3} c_{2}+i_{4} d_{2}, \\
& \alpha=i_{1} a+i_{2} b+i_{3} b+i_{4} d,
\end{aligned}
$$

we find that the coefficient of $i_{1}$ ar the left is

$$
a_{2}\left(a a_{1}+b b_{1}+c c_{1}+d d_{1}\right)-a_{1}\left(a a_{2}+b b_{2}+c c_{2}+d d_{2}\right),
$$

while on the right the coefficient is

$$
-b\left(a_{1} b_{2}\right)+c\left(c_{1} a_{2}\right)-d\left(a_{1} d_{2}\right),
$$

and these are of course identical.

$\S 9$. Let $M$ be at the extremity of the vector $\rho$ and Fig. 1.

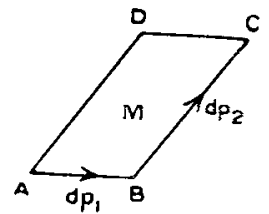

construct about it as centre a small parallelogram bounded by the vectors $d \rho_{1}$ and $d \rho_{2}$ as in the figure. 
If the value of a function be $F(\rho)$ at $M$ then along the side marked $d \rho_{2}$ the value will be

$$
\mathrm{F}(\rho)-\frac{1}{2} \mathrm{~V}_{0} d \rho_{1} \mathrm{D}_{1} . \mathrm{F}(\rho), \ldots . .
$$

in accordance with $\S 6$; while along the opposite side we shall have the value

$$
\mathrm{F}(\rho)+\frac{1}{2} \nabla_{0} d \rho_{1} \mathrm{D}_{1} \cdot \mathrm{F}(\rho), \quad \cdot \quad \cdot \quad .
$$

and there will be similar expressions for the other two sides.

Thus on making the summation $\Sigma d \rho \mathrm{F}(\rho)$ and making the circuit in the positive direction, we find

$$
\begin{aligned}
\Sigma d \rho \mathrm{F}(\rho) & =d \rho_{1} \mathrm{~V}_{0} d \rho_{2} \mathrm{D}_{1} \cdot \mathrm{F}(\rho)-d \rho_{2} \mathrm{~V}_{0} d \rho_{1} \mathrm{D}_{1} \cdot \mathrm{F}(\rho) \\
& =\mathrm{V}_{1}\left(\mathrm{~F}_{2} d \rho_{1} d \rho_{2} \cdot \mathrm{D}_{1}\right) \cdot \mathrm{F}(\rho) \\
& =\mathrm{V}_{1}\left(d \nu \mathrm{D}_{1}\right) \cdot \mathrm{F}(\rho) . \quad . \quad . . . . .
\end{aligned}
$$

If $F(\rho)$ is regular throughout a finite surface we have by adding up for each element of which the surface is composed :

$$
\int d \rho \mathrm{F}(\rho)=\iint \mathrm{V}_{1}\left(d \nu \mathrm{D}_{1}\right) \mathrm{F}(\rho) .
$$

In the summation all the edges of the elementary area except those round the boundary contribute twice to the summation with opposite signs in each case, so that finally the integral is taken round the boundary.

A particular case is obtained by choosing $F(\rho)$ in the form $i_{1} \mathrm{X}+i_{2} \mathrm{Y}+i_{3} \mathrm{Z}+i_{4} \mathrm{U}$, when we obtain :

$$
\begin{aligned}
& \int(\mathrm{X} d x+\mathrm{Y} d y+\mathrm{Z} d z+\mathrm{U} d u) \\
&=\iint\left(\frac{\partial \mathrm{Z}}{\partial y}-\frac{\partial \mathrm{Y}}{\partial z}\right) d y d z+\left(\frac{\partial \mathrm{X}}{\partial z}+\frac{\partial \mathrm{Z}}{\partial x}\right) d z d x \\
&+\left(\frac{\partial \mathrm{Y}}{\partial x}-\frac{\partial \mathrm{X}}{\partial y}\right) d x d y+\left(\frac{\partial \mathrm{U}}{\partial x}-\frac{\partial \mathrm{X}}{\partial u}\right) d x d u \\
&+\left(\frac{\partial \mathrm{U}}{\partial y}-\frac{\partial \mathrm{Y}}{\partial u}\right) d y d u+\left(\frac{\partial \mathrm{U}}{\partial z}-\frac{\partial \mathrm{Z}}{\partial u}\right) d z d u
\end{aligned}
$$

by writing $d \nu$ in the form $(2 \cdot 9)$ and taking the scalar product of each side. 
If $\mathrm{F}$ is of the special form given above since

$$
\begin{aligned}
& d \nu \mathrm{D}_{1}=\mathrm{V}_{3} d \nu \mathrm{D}_{1}+\mathrm{V}_{1} d \nu \mathrm{D}_{1} \\
& \text { and } \\
& \mathrm{V}_{0}\left(\mathrm{~V}_{3} d \nu \mathrm{D}_{1}\right) \cdot \mathrm{F}=0,
\end{aligned}
$$

we have: $\quad V_{0}\left\{V_{1} d v D_{1} . F\right\}=V_{0}\left(d v D_{1} F\right)=V_{0}\left(d \nu V_{2} D_{1} F\right)$,

$$
\therefore \mathrm{V}_{0} \int d \rho \mathrm{F}(\rho)=\iint \mathrm{V}_{0}\left\{\left(\mathrm{~V}_{2} \mathrm{D}_{1} \mathrm{~F}\right) \cdot d \boldsymbol{\nu}\right\}
$$

The curl of a vector is detined by the equation

$$
\mathrm{V}_{0} \int d \rho \mathrm{F}(\rho)=\iint \mathrm{V}_{0}(\operatorname{curl} \mathrm{F} . d \nu) \text {. }
$$

Thus

$$
\text { curl } \mathrm{F}=\mathrm{V}_{2} \mathrm{D}_{1} \mathrm{~F} \text {. }
$$

$\$ 10$. The second theorem obtained by performing the operation $\Sigma d \tau \mathrm{F}(\rho)$ through a sinall four-dimensional element $d v$ connects a three-dimensional to a four-dimensional integral. By a similar treatment in this case as in the last : $\Sigma d \tau \cdot \mathrm{F}(\rho)=$

$$
\begin{aligned}
& -V_{3} d \rho_{2} d \rho_{3} d \rho_{4} \cdot V_{0} d \rho_{1} D_{1} \cdot \mathrm{F}+V_{3} d \rho_{3} d \rho_{4} d \rho_{1} \cdot V_{0} d \rho_{2} D_{1} \cdot \mathrm{F} \\
& -V_{3} d \rho_{4} d \rho_{1} d \rho_{2} . V_{0} d \rho_{3} D_{1} \cdot \mathrm{F}+V_{3} d \rho_{1} d \rho_{2} d \rho_{3} . V_{0} d \rho_{4} D_{1} \cdot \mathrm{F} .
\end{aligned}
$$

This is simplified by observing that :

$$
\begin{aligned}
& V_{4} \alpha_{1} \alpha_{2} \alpha_{3} o_{4} \cdot \alpha=-V_{3} \alpha_{2} \alpha_{3} \alpha_{4} V_{0} \alpha_{1} \alpha+V_{3} \alpha_{3} \alpha_{4} \alpha_{1} V_{0} \alpha_{2} \alpha \\
& -V_{3} \alpha_{4} \alpha_{1} \alpha_{2} \cdot V_{0} \alpha_{3} \alpha+V_{3} \alpha_{1} \alpha_{2} \alpha_{3} . V_{0} \alpha_{4} \alpha . \\
& \therefore \quad \Sigma d \tau, \mathrm{F}(\rho)=\mathrm{V}_{4} d \rho_{1} d \rho_{2} d \rho_{3} d \rho_{4} . \mathrm{D}_{1} \mathrm{~F}(\rho) \\
& =\mathrm{I} d v \cdot \mathrm{D}_{1} \mathrm{~F}(\rho) \text {. } \\
& \therefore \quad \iiint d \tau \cdot \mathrm{F}(\rho)=\iiint \int \mathrm{I} \mathrm{D}_{1} \mathrm{~F}(\rho) \cdot d v, \quad . \\
& \text { or } \iiint \mathrm{I} d \tau \cdot \mathrm{F}(\rho)=\iiint \int \mathrm{D}_{1} \mathrm{~F}(\rho) d v \text {. }
\end{aligned}
$$

The same limitations as before are to be imposed on $\mathrm{F}$ throughout the finite region.

By taking the scalar product of each side and employing again the special form for $\mathrm{F}(\rho)$ we have :

$$
\begin{gathered}
\iiint(\mathrm{X} d y d z d u+\mathrm{Y} d z d u d x+\mathrm{Z} d u d x d y+\mathrm{U} d x d y d z) \\
=\iiint \int\left(\frac{\partial \mathrm{X}}{\partial x^{2}}+\frac{\partial \mathrm{Y}}{\partial y}+\frac{\partial \mathrm{Z}}{\partial z}+\frac{\partial \mathrm{U}}{\partial u}\right) d x d y d z d u .
\end{gathered}
$$

Sommerfeld's extended Div $\mathrm{F}$ is equal to $-\mathrm{V}_{0} \mathrm{D}_{1} \mathrm{~F}$, or to:

$$
\operatorname{Lim}_{d v \rightarrow 0} \frac{-\mathrm{V}_{0} \int \mathrm{I} d \tau \cdot \mathrm{F}(\rho)}{\delta v}
$$


$\$ 11$. The last of the three theorems occupies a place intermediate between the other two and connects an integral in two dimensions with one in three dimensions.

Fig. 2.

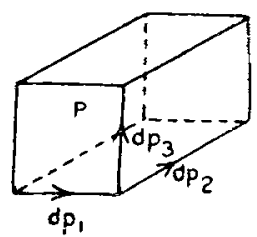

Proceeding as in the other two cases, if $\rho$ defines the centre of the element bounded by the three vectors $d \rho_{1}, d \rho_{2}$, and $d \rho_{3}$ : $\Sigma d \nu \cdot \mathrm{F}(\rho)=$

$$
\begin{aligned}
& V_{2} d \rho_{2} d \rho_{3} \cdot V_{0} d \rho_{1} D_{1} \cdot \mathrm{F}(\rho)-V_{2} d \rho_{3} d \rho_{1} \cdot V_{0} d \rho_{2} D_{1} \cdot \mathrm{F}(\rho) \\
- & V_{2} d \rho_{1} d \rho_{2} \cdot V_{0} d \rho_{3} D_{1} \cdot \mathrm{F}(\rho) .
\end{aligned}
$$

It is easy to show that

$V_{2}\left(V_{3} \alpha_{1} \alpha_{2} \alpha_{3}\right) \cdot \alpha=+V_{2} \alpha_{i} \alpha_{3} \cdot V_{0} \alpha_{1} \alpha+V_{2} \alpha_{3} \alpha_{1} \cdot V_{0} \alpha_{2} \alpha$

so that

$$
+V_{2} \alpha_{1} \alpha_{2} \cdot V_{\beta} \alpha_{3} \alpha_{4}
$$

$$
\begin{aligned}
\sum d \nu . F(\rho) & =-\left\{\mathrm{V}_{2}\left(\mathrm{~V}_{3} d \rho_{1} d \rho_{2} d \rho_{3}\right) \cdot \mathrm{D}_{1}\right\} \mathrm{F}(\rho) \\
& =+\mathrm{V}_{\mathbf{2}}\left(d \tau \mathrm{D}_{1}\right) \cdot \mathrm{F}(\rho) .
\end{aligned}
$$

'Thus by extension as in the previous cases to regions of finite extent :

$$
\iint d \nu \mathrm{F}(\rho)=\iiint \mathrm{V}_{2}\left(d \tau \mathrm{D}_{1}\right) . \mathrm{F}(\rho) ., \quad .
$$

If the summation $\Sigma d \nu^{\prime} F(\rho)$ is required, since

we have:

$$
d \nu^{\prime}=-\mathrm{I} d \nu,
$$

$$
\iint d \nu^{\prime} \mathrm{F}(\rho)=-\iiint I V_{2}\left(d \tau \mathrm{I}_{1}\right) \mathrm{F}(\rho)
$$

Sommerfeld detines the component of the vector divergence normal to $d \tau$ of a six-vector $\mathrm{P}_{2}$ by the operation :

$$
\operatorname{Lim}_{\mathrm{T} \delta \tau \rightarrow 0} \frac{-\int j V_{0}\left(d v^{\prime} \mathrm{P}_{2}\right)}{\mathrm{T} \delta \tau}
$$

where ' $\mathrm{l}$ ' $\delta \tau$ is the magnitude of the three-dimensional volume $\delta \tau$. This expression is called the component normal to $\delta \tau$. 
We may also define Minkowski's lor $P_{2}$ from (11.3), this expression denoting the Lorentz operator, equivalent to the vector divergence.

Lor $\mathrm{P}_{2}$ is defined by the equation :

$$
\begin{aligned}
+\iint \mathrm{V}_{0}\left(d \nu^{\prime} \mathrm{P}_{2}\right) & =-\iiint \mathrm{V}_{0}\left(\operatorname{lor} \mathrm{P}_{2} \cdot \mathrm{I} d \tau\right) \\
& \left.=-\iiint \mathrm{V}_{0} \mathrm{I} \cdot \mathrm{V}_{2} ! d \tau \mathrm{D}_{1}\right) \cdot \mathrm{P}_{2} \\
& =-\iiint \mathrm{V}_{0}\left\{\mathrm{I} d \tau \mathrm{D}_{1} \mathrm{P}_{2}\right\},
\end{aligned}
$$

since the part $\mathrm{V}_{4} d \tau \mathrm{D}_{1}$, which with $\mathrm{V}_{2} d \tau \mathrm{D}_{1}$ makes up the product $d \tau \mathrm{D}_{1}$, cannot add to the scalar product.

Now $\mathrm{I} d \tau$ is a product of the type $\mathrm{V}_{1}$ so that

$$
\begin{array}{rlrl} 
& \mathrm{V}_{0}\left(\mathrm{I} d \tau \cdot \mathrm{D}_{1}, \mathrm{P}_{2}\right) & =\mathrm{V}_{0}\left(\mathrm{I} d \tau \cdot \mathrm{V}_{1} \mathrm{D}_{1} \mathrm{P}_{2}\right), \\
\therefore & & \text { lor } \mathrm{P}_{2} & =\mathrm{V}_{1} \mathrm{D}_{1} \mathrm{P}_{2} *
\end{array}
$$

Since by $\S 4, \mathrm{~V}_{0}\left(d \nu \mathrm{P}_{2}\right)=\mathrm{V}_{0}\left(d \nu^{\prime} \mathrm{P}_{2}^{\prime}\right)$, we have by $(12 \cdot 4)$ and $(12 \cdot 2)$

$\iint \mathrm{V}_{0}\left(d \nu \mathrm{P}_{2}\right)=\iint \mathrm{V}_{0}\left(d \nu^{\prime} \mathrm{P}_{2}^{\prime}\right)=-\iiint \mathrm{V}_{0}\left(\operatorname{lor} \mathrm{P}_{2}^{\prime} . \mathrm{I} d \tau\right)$.

Thus in integrating the normal components of $\mathrm{P}_{2}$ over a surface, the operation lor $\mathrm{P}_{2}$ occurs, while for tangential components (i.e. components of $\mathrm{P}_{2}$ along $d v$ ) lor $\mathrm{P}_{2}$ is replaced by $\operatorname{lor} \mathrm{P}_{2}^{\prime}$.

This is important in the electromagnetic equations.

\$12. The field equations of the electron theory as expressed by Minkowski may now be regarded as a generalization of Gauss's integral of intensity over a surface.

Putting these integrals in their usual form we have:

$$
\begin{array}{ll}
\int \mathrm{D}_{n} d \mathrm{~S}=-\int \rho d v & \text { or } \int \mathrm{S} \mathrm{D} d \nu=\int \rho d v, \\
\int \mathrm{B}_{n} d \mathrm{~S}=0 & \text { or. } \int \mathrm{SB} d \nu=0 .
\end{array}
$$

If now we form two six-vectors :

$$
\begin{aligned}
\mathrm{P}_{2}=\left(-i \mathrm{D}_{x}\right) i_{2} i_{3}+\left(-i \mathrm{D}_{y}\right) i_{3} i_{1}+\left(-i \mathrm{D}_{z}\right) i_{1} i_{2} & +\mathrm{H}_{x} i_{1} i_{4} \\
& +\mathrm{H}_{y} i_{2} i_{4} \\
& +\mathrm{H}_{z} i_{3} i_{4}, \\
\mathrm{Q}_{2}=i \mathrm{~B}_{x} i_{2} i_{3}+\ldots & +\mathrm{E}_{x} i_{1} i_{4}+\ldots .
\end{aligned}
$$

where $i=\sqrt{-1}$. $\quad \mathrm{D}_{x}$ etc. denote the components of displacement as usual, and $\mathrm{H}, \mathrm{B}$, and $\mathrm{E}$ have their usual significance.

* Cf. Cunningham, 'Theory of Relativity,' pp. 101-2. 
If $S$ is the current four-vector and equal to

$$
\rho u_{x} i_{1}+\rho u_{y} i_{2}+\rho u_{z} i_{3}+\rho i i_{4},
$$

where $\rho$ is the density of charge and the $u$ 's components of velocity of the charge, the general equations may then be written :

$$
\begin{aligned}
& \text { Lor } \mathrm{P}_{2}=\mathrm{S}, \\
& \text { Lor } \mathrm{Q}_{2}=0 \text {. }
\end{aligned}
$$

and for the case when the permeability and dielectric constant are both unity $\mathrm{Q}_{2}=\mathrm{P}_{2}^{\prime}$.

Thus from (11.4) and (11.5) we may summarize the theory by stating that the normal component of the six-vector $\mathrm{P}_{2}$ over the surface of a three-dimensional volume is numerically equal to the amount of $\mathbb{S}$ within the volume, while the total tangential component vanishes over the surface. By the amount of $S$ is meant the numerical value of

$$
\mathrm{V}_{0} \int \mathrm{I} d \tau \cdot \mathrm{S} \text {. }
$$

$\mathrm{It}$ is the value of the component of $\mathrm{S}$ along the four-vector $\mathrm{I} d \tau$.

$\$ 13$. The four-vectors of Minkowski's Calculus are limited in that they are subject to a linear transformation. The vector $\rho$ becomes a new vector $\sigma$ where

$$
\sigma=\phi \rho \text {. }
$$

$\phi$ is a linear vector operator and can be expressed as :

$$
\phi \rho=-\left(\alpha_{1} \mathrm{~V}_{0} \alpha_{2} \rho+\beta_{1} \mathrm{~V}_{0} \beta_{2} \rho+\gamma_{1} \mathrm{~V}_{0} \gamma_{2} \rho+\delta_{1} \mathrm{~V}_{0} \delta_{2} \rho\right) \text {. }
$$

Thus $\phi$ is a dyadic and associated with it are certain invariants just as in the case of three dimensions, and whereas in the simpler case $\phi$ satisfies a certain cubic relation so here $\phi$ satisfies a quartic.

In three dimensions the ratio of the two scalar products $\mathrm{S} \phi \lambda \phi \mu \phi \nu$ to $\mathrm{S} \lambda \mu \nu$ is independent of $\lambda, \mu$, and $\nu$; to this corresponds the invariance of the ratio $\mathrm{TV}_{4} \phi \lambda \phi \mu \phi \nu \phi \pi$ to $\mathrm{TV}_{4} \lambda \mu \nu \pi$, which means that the ratios of the four-dimensional volumes after and before the operation of $\phi$ are a constant. When $\phi$ denotes the Einstein transformations this ratio is unity.

The linear transformation referred to is analogous to that occurring in the theory of elasticity in the case of homogeneous strain, and since a further condition to be satisfied by the four-vector is that of unchanging tensor such a transformation is analogous to a rotation. 
404 Integration Theorems of Four-Dimensional Analysis.

Gibbs has shown that a rotation may be expressed by

$$
\phi \rho=i^{\prime}(i \rho)+j^{\prime}(j \rho)+k^{\prime}(k \rho),
$$

where $(i \rho)=$ the scalar product of $i$ and $\rho$; and we may also express a rotation in four-vectors similarly,

$$
\phi \rho=-\left(\alpha^{\prime} \mathrm{V}_{0} \alpha \rho+\beta^{\prime} \mathrm{V}_{0} \beta \rho+\gamma^{\prime} \mathrm{V}_{0} \gamma \rho+\delta^{\prime} \mathrm{V}_{0} \delta \rho\right),
$$

$\imath^{\prime} j^{\prime} k^{\prime}, i j k$, are unit vectors and each group is mutually perpendicular. Similarly $\alpha^{\prime} \beta^{\prime} \gamma^{\prime} \delta^{\prime}$ and $\alpha \beta \gamma \delta$ are unit mutually perpendicular vectors.

The Einstein transformation is a particular case of this in which

$$
\begin{aligned}
& \alpha^{\prime}=k\left(i_{1}-i_{v} i_{4}\right), \quad \alpha=i_{1}, \\
& \beta=i_{2}=\beta^{\prime}, \\
& \gamma=i_{3}=\gamma^{\prime}, \\
& \delta^{\prime}=k\left(i v i_{1}+i_{4}\right),
\end{aligned}
$$

where $k=\left(1-v^{2}\right)^{-\frac{1}{2}}, i=\sqrt{-1} ; v$ the arbitrary constant in $\phi$ is the velocity and may be said to define the strain.

The notation suggests that the restricted principle of Relativity may be summed up by stating that a fundamental four-dimensional medium exists which may be subjected to a strain of the type $(13 \cdot 1)$ with the values of the vectors given above and that phenomena described by the unstrained vectors will be described by the strained vectors in exactly the same way.

It is natural to generalize $\phi$ and give up the demand that it should be line:r.

The strain is then heterogeneous and the principle becomes more general.

For small regions there is still a linear relation, for if $\sigma$ is a function of $\rho$ we have :

$$
d \sigma=-\mathrm{V}_{0} d \rho \mathrm{D}_{1}, \sigma=\phi d \rho ;
$$

but $\phi$ now contains $\rho$ in its constitution and the linear relation is truo only for small changes $d \rho$ in the neighhourhood of $\rho$.

This appears to correspond to the "naturalness" of small regions in the theory of Relativity. Space-time is Galilean for infinitesimal regions. In addition $(d \sigma)^{2}$ is invariant or

$$
(d \sigma)^{2}=(d \rho)^{2} .
$$

